\section{Barreiras de acesso aos serviços em cinco Regiões de Saúde do Brasil: percepção de gestores e profissionais do Sistema Único de Saúde}

\author{
Barriers in access to services in five Health \\ Regions of Brazil: perceptions of policymakers \\ and professionals in the Brazilian Unified \\ National Health System
}

\section{Barreras de acceso a servicios en cinco Regiones de Salud de Brasil: percepción de gestores y profesionales del Sistema Único de Salud}

Ricardo Antunes Dantas de Oliveira 1 Cristina Maria Rabelais Duarte 1,2 Ana Luiza Braz Pavão 1 Francisco Viacava 1

doi: 10.1590/0102-311X00120718

\title{
Resumo
}

O objetivo foi analisar as barreiras de acesso aos serviços de saúde existentes em cinco Regiões de Saúde do Brasil. Foram analisadas as respostas obtidas a partir da aplicação de questionários semiestruturados a gestores, prestadores e, em número mais restrito, a profissionais de saúde do Sistema Único de Saúde, tendo como referência as dimensões: Acessibilidade Geográfica, Disponibilidade e Aceitabilidade. Foram verificadas as especificidades em relação às barreiras de acesso nas regiões consideradas, já outras como a localização e tempo gasto no trajeto foram barreiras em quase todas elas quando foi considerado o cuidado ao acidente vascular cerebral. Quando à Disponibilidade, foram observadas questões relacionadas à insuficiência de médicos, tempo de espera prolongado e problemas relativos à integração, em todas as regiões. Quanto à Aceitabilidade, destacou-se a crença dos usuários de não possuir problemas de saúde. As Regiões de Saúde situadas no Sul e Sudeste do país registraram menor quantidade e diversidade de barreiras, revelando a persistência de desigualdades regionais. Embora os resultados não representem o conjunto do Brasil, permitem apontar questões relevantes sobre o processo de regionalização do sistema de saúde no país.

Acesso aos Serviços de Saúde; Regionalização; Desigualdades em Saúde

\author{
Correspondência \\ R. A. D. Oliveira \\ Instituto de Comunicação e Informação Científica e Tecnológica \\ em Saúde, Fundação Oswaldo Cruz. \\ Av. Brasil 4036, sala 713, Rio de Janeiro, RJ 21041-361, Brasil. \\ ricardo.dantas@icict.fiocruz.br

\footnotetext{
1 Instituto de Comunicação e Informação Científica e Tecnológica em Saúde, Fundação Oswaldo Cruz, Rio de Janeiro, Brasil.

2 Faculdade de Medicina de Petrópolis, Petrópolis, Brasil.
} 


\section{Introdução}

A constituição de uma rede regionalizada e hierarquizada de serviços de saúde tem sido um dos principais desafios ao longo dos 30 anos do Sistema Único de Saúde (SUS) 1. A regionalização requer arranjos institucionais peculiares, tendo em vista que a criação de regiões depende da pactuação federativa entre o governo estadual e os governos dos municípios que as compõem, e também de acordos horizontais entre os Secretários Municipais, que extrapolam os mecanismos contratuais que vigoram em consórcios municipais. Até o presente momento, a regionalização deriva do espírito de cooperação do "federalismo sanitário brasileiro" 2 .

O Governo Federal, com base em resoluções da Comissão Intergestores Tripartite (CIT), publicou, em 2011, o Decreto no 7.508/20113 que regulamenta a Lei no 8.080/1990 e estabelece a estrutura organizativa do SUS, o planejamento em saúde, a assistência à saúde e a articulação interfederativa. Um de seus aspectos está relacionado ao acesso às ações e serviços de saúde, que deve ser ordenado pela atenção primária, completando-se na rede regionalizada e hierarquizada, de acordo com a complexidade do serviço. Apesar do arcabouço jurídico-legal, é reconhecida a persistência de diversos tipos de barreiras que interferem na concretização do acesso universal, preconizado como um dos princípios do SUS 4,5,6.

Embora o acesso à assistência à saúde seja frequentemente apresentado como um objetivo da política de saúde, seu conceito apresenta grande diversidade na literatura 7,8 , variando entre autores e com o contexto, mudando ao longo do tempo e empregando distintas terminologias 9 . De acordo com a Joint Commission on Accreditation of Healthcare Organizations (JCAHO) 10, acesso diz respeito ao grau com que o cuidado e a intervenção adequados estão disponíveis para responder às necessidades dos pacientes. Essa definição também é assumida pelo Observatório Europeu de Políticas e Sistemas de Saúde 11, cujos integrantes defendem que o acesso a serviços de saúde constitui precondição de cidadania, sendo consensual que sua distribuição obedeça às necessidades de saúde. Esse conceito reconhece a necessidade de interação entre sistemas de saúde e indivíduos, famílias e comunidades onde vivem 7,8 .

Para avaliar a qualidade do acesso, a literatura apresenta diversas categorias entre as quais são comuns a acessibilidade geográfica, disponibilidade, viabilidade e aceitabilidade. Em cada uma delas há um conjunto de barreiras, distribuído entre oferta e demanda por serviços de saúde 10,12,13. A acessibilidade geográfica diz respeito à adequação da localização dos serviços de saúde em relação à localização dos usuários, levando em conta distância, meios de transporte e tempo de deslocamento. A disponibilidade reflete a existência dos serviços e recursos de saúde em quantidade e qualidade apropriadas às necessidades dos usuários e a maneira pela qual os recursos são organizados para atender aos usuários. A viabilidade é o grau de adequação entre o custo da utilização dos serviços de saúde e a capacidade de pagamento dos indivíduos. A aceitabilidade diz respeito a percepções e atitudes dos usuários em relação a profissionais e práticas, e à percepção e atitudes dos profissionais e provedores a respeito das características pessoais dos usuários 13.

Para dar seguimento às definições do Decreto no 7.508/2011, ganha especial relevo a avaliação de experiências de regionalização, de modo a favorecer o processo decisório da gestão do SUS, sendo este o contexto no qual está inserido o presente artigo, cujo objetivo é avaliar as barreiras de acesso existentes em cinco Regiões de Saúde localizadas nas diferentes grandes regiões geográficas brasileiras, com base nas visões de gestores, prestadores e profissionais de saúde. O estudo oferece uma perspectiva das regiões de saúde em diferentes cenários, possibilitando a identificação da diversidade de barreiras existentes em cada contexto, de modo a subsidiar o planejamento de ações que visem à ampliação do acesso.

\section{Métodos}

Estudo de natureza descritiva, desenvolvido com base em dados qualitativos selecionados de entrevistas semiestruturadas realizadas no estudo de campo do projeto multicêntrico de pesquisa Região $e$ Redes - Política, Planejamento e Gestão das Regiões e Redes de Atenção à Saúde no Brasil 14, cujo objetivo foi avaliar processos de organização, coordenação e gestão envolvidos na conformação de regiões e redes de atenção à saúde no SUS. 
O estudo de campo do projeto multicêntrico foi realizado em cinco Regiões de Saúde: Norte e Sul de Barretos (São Paulo); Petrolina (Pernambuco)/Juazeiro (Bahia); Manaus, Entorno e Alto Rio Negro (Amazonas); Baixada Cuiabana (Mato Grosso); e Carbonífera/Costa Doce (Rio Grande do Sul), entre agosto de 2015 e junho de 2016. As Regiões de Saúde foram escolhidas de modo a valorizar a variabilidade presente nos territórios: contemplam populações de 384.175 a 2.119.145 habitantes, com variadas densidades demográficas, taxas de crescimento e envelhecimento populacional. Incluem uma metrópole nacional, uma capital regional, municípios de diferentes redes urbanas, áreas de fronteira estadual e internacional, e regiões com diferentes composições de serviços públicos e privados 14 . A opção por tal diversidade desaconselha a comparação direta dos resultados deste trabalho, oferecendo, por outro lado, a perspectiva de revelar barreiras de acesso presentes em cenários com composições de características bastante específicas.

As variáveis do presente estudo correspondem a itens estruturados, originados de roteiros de entrevistas realizadas, no âmbito do projeto multicêntrico, com gestores e prestadores de serviço dos níveis municipal, estadual e regional, além de gestores ou profissionais - médicos e enfermeiros - vinculados especificamente à rede de urgência e emergência ou à vigilância em saúde, cuja seleção ocorreu em função da familiaridade do respondente com a temática da entrevista. Os roteiros, específicos para cada tipo de respondente e temática, incluíram respostas categorizadas em escala nominal (sim, não/não sabe) ou ordinal (sempre, quase sempre, às vezes, raramente, nunca/não sabe). $\mathrm{O}$ número de respondentes variou do mínimo de 24 ao máximo de 105, com a maioria concentrada entre 50 e 80 respondentes.

O Quadro 1 traz as dimensões e barreiras de acesso definidas com base no referencial teórico-conceitual adotado 13, adaptadas em função das variáveis selecionadas do referido projeto multicêntrico de pesquisa, e no tipo de resposta (nominal ou ordinal). Como sinalizado no Quadro 1, os aspectos avaliados podem dizer respeito a diferentes níveis de atenção, a toda a rede de assistência em saúde (RAS) ou referidos à rede de cuidado ao acidente vascular cerebral (AVC), um dos eventos traçadores utilizados no referido projeto de pesquisa.

No caso dos itens com escala nominal, considerou-se a manifestação de pelo menos metade dos respondentes $(50 \%)$ como indicativa da existência de barreiras relevantes. No caso dos itens com escala ordinal foram usados os escores médios, com valores entre 1 e 5 , onde 1 equivale à pior avaliação e 5, à melhor avaliação. Foram considerados indicativos de barreiras relevantes escores médios superiores a 3 .

Os resultados estão organizados em três seções de acordo com as dimensões de acesso apresentadas, sendo analisados no contexto das próprias regiões consideradas, buscando evidenciar distinções nas realidades regionais.

\section{Resultados}

\section{Acessibilidade Geográfica}

O Quadro 2 traz as questões referentes às barreiras de acesso relativas à Acessibilidade Geográfica. O tempo de deslocamento superior a duas horas para a realização de consultas se destaca em duas regiões de grande área territorial: Petrolina/Juazeiro e Manaus, Entorno e Alto Rio Negro, configurando-se como uma barreira relevante nestes contextos.

Em relação à atenção ao AVC, observou-se que o tempo gasto no trajeto é barreira em quase todas as regiões, diferindo de acordo com as unidades sobre as quais se referem nos vários contextos. Já a questão da segurança e preocupação com a violência, é marcante na Região da Baixada Cuiabana, incluindo quase todas as unidades prestadoras.

Considerando a demanda, observou-se que os entrevistados das regiões Carbonífera/Costa Doce e Barretos não apontaram o custo dos meios de transporte como uma barreira de acesso a nenhum dos tipos de serviços considerados no cuidado ao AVC. Nas regiões de Petrolina/Juazeiro e Baixada Cuiabana, ao contrário, para todos os serviços, o custo dos transportes foi considerado uma barreira para os pacientes. Na Região de Manaus, destacou-se como uma barreira relevante para a maioria dos serviços. 
Quadro 1

Variáveis selecionadas do estudo de campo do projeto multicêntrico Região e Redes - Política, Planejamento e Gestão das Regiões e Redes de Atenção à Saúde no Brasil, realizado entre 2015 e 2016, segundo dimensões e barreiras de acesso.

\begin{tabular}{|c|c|c|c|}
\hline \multicolumn{4}{|c|}{ DIMENSÃO: ACESSIBILIDADE GEOGRÁFICA } \\
\hline \multirow{3}{*}{ Oferta } & \multirow{3}{*}{ Localização dos serviços } & $\begin{array}{l}\text { Tempo de deslocamento superior a } 2 \text { horas para consultas especializadas de } \\
\text { cardiologia, neurologia e de reabilitação. }\end{array}$ & \multirow{3}{*}{$\mathrm{O}$} \\
\hline & & $\begin{array}{l}\text { Tempo gasto no trajeto de busca por cuidado ao AVC em unidades de urgência } \\
\text { e emergência, internação, tratamento intensivo, de diagnóstico por imagem e de } \\
\text { reabilitação. }\end{array}$ & \\
\hline & & $\begin{array}{l}\text { Segurança no entorno da unidade e preocupação com violência na busca de } \\
\text { cuidado ao AVC, em unidades de urgência e emergência, internação, tratamento } \\
\text { intensivo, de diagnóstico por imagem e de reabilitação. }\end{array}$ & \\
\hline Demanda & Custo dos transportes & $\begin{array}{l}\text { Custo dos meios de transportes na busca por cuidado ao AVC em unidades de } \\
\text { urgência e emergência, internação, tratamento intensivo, de diagnóstico por } \\
\text { imagem e de reabilitação. }\end{array}$ & $\mathrm{N}$ \\
\hline \multicolumn{4}{|c|}{ DIMENSÃO: DISPONIBILIDADE } \\
\hline \multirow{15}{*}{ Oferta } & \multirow{5}{*}{ Falta de oportunidades } & $\begin{array}{l}\text { Pouca oferta de consultas especializadas de cardiologia, neurologia e de } \\
\text { reabilitação na própria região. }\end{array}$ & $\mathrm{O}$ \\
\hline & & $\begin{array}{l}\text { Indisponibilidade de serviços na RAS (cobertura da atenção primária em saúde, } \\
\text { consultas especializadas, leitos gerais, leitos para procedimentos eletivos, } \\
\text { neurocirurgia e cirurgia cardíaca, UTI adulto, UTI neonatal, SADT de média } \\
\text { complexidade, SADT de alta complexidade). }\end{array}$ & \multirow[t]{3}{*}{$\mathrm{N}$} \\
\hline & & Indisponibilidade de médicos na RAS. & \\
\hline & & Indisponibilidade de enfermeiros na RAS. & \\
\hline & & Outras situações relacionadas à indisponibilidade na RAS. & $\mathrm{O}$ \\
\hline & \multirow{4}{*}{ Integração } & Falta de integração entre serviços na RAS. & \multirow{4}{*}{$\mathrm{N}$} \\
\hline & & $\begin{array}{l}\text { Inexistência de fluxos assistenciais definidos pela CIR para a atenção especializada } \\
\text { e hospitalar na RAS. }\end{array}$ & \\
\hline & & Percurso da atenção primaária à saúde para consulta especializada indefinido. & \\
\hline & & $\begin{array}{l}\text { Ausência de compartilhamento de responsabilidade e fluxo de informações em } \\
\text { tempo oportuno no cuidado ao AVC. }\end{array}$ & \\
\hline & \multirow[b]{3}{*}{ Tempo de espera } & Lista de espera com tempo inadequado às necessidades. & \multirow[b]{3}{*}{$\mathrm{O}$} \\
\hline & & Tempo de espera incompatível com o atendimento de Emergência. & \\
\hline & & $\begin{array}{l}\text { Agendamento de serviços em tempo oportuno (consultas em cardiologia, } \\
\text { neurologia, oftalmologia, endocrinologia, nefrologia, nutrição e em reabilitação; } \\
\text { ECG, ecocardiograma, exame de fundo de olho, perfil lipídico, mamografia } \\
\text { (mulheres de } 50 \text { a } 69 \text { anos), colposcopia). }\end{array}$ & \\
\hline & Falta de Medicamentos & Insuficiência de medicamentos. & $\mathrm{O}$ \\
\hline & Horário de funcionamento & $\begin{array}{l}\text { Horário de funcionamento inadequado, em relação ao cuidado ao AVC, de } \\
\text { unidades de urgência e emergência, diagnóstico por imagem e de reabilitação. }\end{array}$ & $\mathrm{N}$ \\
\hline & Qualificação dos profissionais & $\begin{array}{l}\text { Qualificação insuficiente dos profissionais no cuidado ao AVC em unidades de } \\
\text { urgência e emergência, internação, tratamento intensivo, de diagnóstico por } \\
\text { imagem e de reabilitação. }\end{array}$ & $\mathrm{N}$ \\
\hline Demanda & Informação sobre os serviços de saúde & Incapacidade de identificar local apropriado para receber atendimento. & $\mathrm{N}$ \\
\hline \multicolumn{4}{|c|}{ DIMENSÃO: ACEITABILIDADE } \\
\hline Oferta & Acolhimento pelos profissionais & $\begin{array}{l}\text { Forma de receber os pacientes para o cuidado ao AVC em unidades de urgência } \\
\text { e emergência, internação, tratamento intensivo, de diagnóstico por imagem e de } \\
\text { reabilitação. }\end{array}$ & $\mathrm{N}$ \\
\hline \multirow{3}{*}{ Demanda } & Preferências culturais e comunitárias & Preferência por formas alternativas de cuidados. & $\mathrm{N}$ \\
\hline & Crença de não ter problema de saúde & Crença de não ter problema de saúde. & $\mathrm{N}$ \\
\hline & Estigma & $\begin{array}{l}\text { Estigma: preocupação com o julgamento do grupo social a respeito da busca pelo } \\
\text { cuidado. }\end{array}$ & $\mathrm{N}$ \\
\hline
\end{tabular}

AVC: acidente vascular cerebral; CIR: Comissão Intergestores Regionais; ECG: eletrocardiograma; N: escala nominal; O: escala ordinal; RAS: rede de assistência em saúde; SADT: Serviço de Apoio Diagnóstico e Terapêutico; UTI: unidade de tratamento intensivo.

Fonte: tradução livre e adaptação de Jacobs et al. ${ }^{13}$. 


\section{Quadro 2}

Barreiras de acesso referentes à Acessibilidade Geográfica, por parte da oferta e da demanda, segundo tipo de barreira e Região de Saúde, 2016.

\begin{tabular}{|c|c|c|c|c|c|}
\hline Barreiras & $\begin{array}{c}\text { Baixada } \\
\text { Cuiabana (Mato } \\
\text { Grosso) }\end{array}$ & $\begin{array}{l}\text { Manaus, } \\
\text { Entorno e Alto } \\
\text { do Rio Negro } \\
\text { (Amazonas) }\end{array}$ & $\begin{array}{c}\text { Petrolina } \\
\text { (Pernambuco)/ } \\
\text { Juazeiro (Bahia) }\end{array}$ & $\begin{array}{c}\text { Carbonífera/Costa } \\
\text { Doce (Rio Grande } \\
\text { do Sul) }\end{array}$ & $\begin{array}{c}\text { Norte-Barretos/ } \\
\text { Sul-Barretos (São } \\
\text { Paulo) }\end{array}$ \\
\hline \multicolumn{6}{|l|}{ OFERTA } \\
\hline $\begin{array}{l}\text { Tempo de deslocamento } \\
\text { superior a } 2 \text { horas para } \\
\text { consultas }\end{array}$ & Não & Sim & Sim & Não & Não \\
\hline $\begin{array}{l}\text { Tempo gasto no trajeto } \\
\text { para o cuidado ao AVC * }\end{array}$ & $\begin{array}{l}\text { Sim, a não ser } \\
\text { nas unidades de } \\
\text { internação }\end{array}$ & $\begin{array}{l}\text { Sim nas UTIs e } \\
\text { laboratórios/ } \\
\text { unidades de } \\
\text { diagnóstico por } \\
\text { imagem }\end{array}$ & $\begin{array}{l}\text { Sim nas UTIs e de } \\
\text { reabilitação e nos } \\
\text { laboratórios/unidades } \\
\text { de diagnóstico por } \\
\text { imagem }\end{array}$ & $\begin{array}{l}\text { Sim nas UTIs } \\
\text { e unidades de } \\
\text { internação }\end{array}$ & Não \\
\hline $\begin{array}{l}\text { Segurança no entorno da } \\
\text { unidade e a preocupação } \\
\text { com a violência no } \\
\text { cuidado ao AVC * }\end{array}$ & $\begin{array}{l}\text { Sim, a não ser } \\
\text { nas unidades de } \\
\text { internação }\end{array}$ & Não & Não & Não & Não \\
\hline \multicolumn{6}{|l|}{ DEMANDA } \\
\hline $\begin{array}{l}\text { Custo dos meios de } \\
\text { transportes no cuidado } \\
\text { ao AVC * }\end{array}$ & $\begin{array}{l}\text { Sim em todos os } \\
\text { tipos de unidades }\end{array}$ & $\begin{array}{l}\text { Sim, a não ser } \\
\text { nas unidades } \\
\text { de internação e } \\
\text { reabilitação }\end{array}$ & $\begin{array}{c}\text { Sim em todos os tipos } \\
\text { de unidades }\end{array}$ & Não & Não \\
\hline
\end{tabular}

AVC: acidente vascular cerebral; UTI: unidade de tratamento intensivo.

Fonte: estudo de campo da pesquisa Região e Redes - Política, Planejamento e Gestão das Regiões e Redes de Atenção à Saúde no Brasil 14.

* Unidades de urgência e emergência, internação, tratamento intensivo e reabilitação, e laboratórios e unidades de diagnóstico por imagem.

As barreiras de acesso relacionadas à Acessibilidade Geográfica são marcantes em três das regiões consideradas: Baixada Cuiabana, Manaus e Petrolina/Juazeiro. Em Barretos, esse tipo de barreira não apresentou destaque em nenhum dos aspectos considerados, já na Região Carbonífera/Costa Doce, o tempo gasto no trajeto é barreira no cuidado ao AVC.

\section{Disponibilidade}

A primeira das questões relativas à Disponibilidade diz respeito à "falta de oportunidades" de atenção à saúde vinculada à indisponibilidade de serviços e profissionais (Quadro 3). A importância da barreira referente à realização de consultas especializadas específicas na própria Região de Saúde é observada em relação à neurologia na Região Carbonífera/Costa Doce. Essa é caracterizada pela realização de consultas em outra Região de Saúde, possivelmente a de Porto Alegre, que está a cerca de 30km do maior município da região, Guaíba.

Os entrevistados destacaram insuficiência generalizada em quase todas as regiões. A cobertura da atenção primária é suficiente apenas nas regiões de Petrolina/Juazeiro e Barretos, onde três serviços foram destacados como suficientes para atender à população.

Quanto aos profissionais, há uma insuficiência generalizada de médicos e suficiência de enfermeiros em quase todas as regiões. Considerando outras situações relacionadas à indisponibilidade 
Quadro 3

Barreiras de acesso referentes à Disponibilidade, por parte da oferta, segundo tipo de barreira e Região de Saúde, 2016.

\begin{tabular}{|c|c|c|c|c|c|}
\hline Barreiras & $\begin{array}{c}\text { Baixada Cuiabana } \\
\text { (Mato Grosso) }\end{array}$ & $\begin{array}{l}\text { Manaus, } \\
\text { Entorno e Alto } \\
\text { do Rio Negro } \\
\text { (Amazonas) }\end{array}$ & $\begin{array}{c}\text { Petrolina } \\
\text { (Pernambuco)/ } \\
\text { Juazeiro (Bahia) }\end{array}$ & $\begin{array}{l}\text { Carbonífera/ } \\
\text { Costa Doce (Rio } \\
\text { Grande do Sul) }\end{array}$ & $\begin{array}{l}\text { Norte-Barretos/ Sul- } \\
\text { Barretos (São Paulo) }\end{array}$ \\
\hline \multicolumn{6}{|l|}{ OFERTA } \\
\hline $\begin{array}{l}\text { Pouca oferta de consultas } \\
\text { (cardiologia, neurologia } \\
\text { e reabilitação) na própria } \\
\text { região }\end{array}$ & Não & Não & Não & Sim em neurologia & Não \\
\hline $\begin{array}{l}\text { Indisponibilidade de } \\
\text { serviços na RAS * }\end{array}$ & Sim & Sim & $\begin{array}{l}\text { Não para atenção } \\
\text { primária à saúde/ } \\
\text { Sim para outros }\end{array}$ & Sim & $\begin{array}{l}\text { Não para atenção } \\
\text { primária à saúde, } \\
\text { leitos gerais e } \\
\text { SADT (média } \\
\text { complexidade)/Sim } \\
\text { para outros }\end{array}$ \\
\hline $\begin{array}{l}\text { Indisponibilidade de } \\
\text { médicos na RAS }\end{array}$ & Sim & Sim & Sim & Sim & Sim \\
\hline $\begin{array}{l}\text { Indisponibilidade de } \\
\text { enfermeiros na RAS }\end{array}$ & Não & Não & Não & Sim & Não \\
\hline $\begin{array}{l}\text { Outras situações } \\
\text { relacionadas à } \\
\text { indisponibilidade }\end{array}$ & $\begin{array}{c}\text { Barreiras de } \\
\text { acesso para PCDs } \\
\text { no cuidado ao } \\
\text { AVC/ Insuficiência } \\
\text { de vagas }\end{array}$ & Não & $\begin{array}{c}\text { Migração de } \\
\text { usuários para } \\
\text { outras regiões / } \\
\text { Insuficiência de } \\
\text { vagas }\end{array}$ & $\begin{array}{c}\text { Migração de } \\
\text { usuários para } \\
\text { outras regiões/ } \\
\text { Insuficiência de } \\
\text { vagas }\end{array}$ & $\begin{array}{l}\text { Migração de usuários } \\
\text { para outras regiões/ } \\
\text { Insuficiência de vagas }\end{array}$ \\
\hline $\begin{array}{l}\text { Falta de integração entre } \\
\text { serviços na RAS }\end{array}$ & Sim & Sim & Não & Não & Não \\
\hline $\begin{array}{l}\text { Inexistência de fluxos } \\
\text { assistenciais definidos } \\
\text { pela CIR }\end{array}$ & Sim & Sim & Não & Não & Não \\
\hline $\begin{array}{l}\text { Percursos da atenção } \\
\text { primária à saúde para } \\
\text { consulta indefinidos }\end{array}$ & Sim & Não & Sim & Sim & Não \\
\hline $\begin{array}{l}\text { Ausência de } \\
\text { compartilhamento de } \\
\text { responsabilidades e fluxo } \\
\text { de informações em tempo } \\
\text { oportuno no cuidado ao } \\
\text { AVC }\end{array}$ & Sim & Sim & Sim & Sim & Sim \\
\hline
\end{tabular}

AVC: acidente vascular cerebral; CIR: Comissão Intergestores Regionais; PCDs: pessoas com deficiências; RAS: rede de assistência em saúde; SADT: Serviço de Apoio Diagnóstico e Terapêutico; UTI: unidade de tratamento intensivo.

Fonte: estudo de campo da pesquisa Região e Redes - Política, Planejamento e Gestão das Regiões e Redes de Atenção à Saúde no Brasil 14.

* Cobertura da atenção primária à saúde, consultas especializadas, leitos gerais, leitos para procedimentos eletivos, neurocirurgia e cirurgia cardíaca,

UTI adulto, UTI neonatal, SADT de média complexidade, SADT de alta complexidade. 
de serviços, observa-se que há uma percepção generalizada de insuficiência de vagas de internação, que possivelmente está relacionada à migração de usuários para outras regiões em busca de atenção à saúde. A Baixada Cuiabana foi a única que se caracterizou pela insuficiência de serviços no cuidado ao AVC.

A integração entre os serviços configura-se como barreira de acesso quando não existe ou é falha. A integração em geral e a existência de fluxos definidos pela Comissão Intergestores Regionais (CIR) são reduzidas nas Regiões de Saúde localizadas no Centro-oeste e no Norte, o que as opõe às demais. Esse padrão não se repete quando considerado o percurso da atenção primária para as consultas especializadas, já que nas Regiões de Saúde de Manaus e Baixada Cuiabana, além da Região Carbonífera/ Costa Doce, a consulta é agendada pela própria atenção primária. Nas outras, não há um percurso definido. É importante frisar que o compartilhamento de responsabilidades e fluxos de informações em tempo oportuno no cuidado ao AVC é percebido como ausente em todas as regiões.

No Quadro 4, observa-se que o tempo de espera também foi abordado baseando-se em três aspectos: lista de espera com tempo inadequado às necessidades, tempo de espera incompatível com o atendimento em urgência/emergência e agendamento de serviços em tempo oportuno. As duas primeiras estão presentes em todas as regiões estudadas. Por outro lado, quanto ao agendamento, destaca-se que ocorre em tempo inoportuno para as consultas na Baixada Cuiabana e para a maioria dos serviços na Região de Manaus. Nas Regiões de Saúde com melhores condições, foram registrados problemas com relação ao agendamento no caso de consultas de neurologia e endocrinologia, além dos exames de eletrocardiograma e ecocardiograma na Região Carbonífera/Costa Doce. A insuficiência de medicamentos é considerada barreira de acesso nas Regiões de Saúde de Barretos e Petrolina/Juazeiro.

Os dois últimos aspectos, horário de funcionamento e qualificação dos profissionais, foram construídos em relação ao cuidado ao AVC em cinco tipos de unidades. Ambos não foram destacados nas regiões Carbonífera/Costa Doce e Barretos. Na Região de Petrolina/Juazeiro, o horário de funcionamento dificulta o acesso a unidades/laboratório de diagnóstico por imagem e, na Região de Manaus, a qualificação de profissionais é um problema nas unidades de Urgência e Emergência. Ambos constituem barreiras na Baixada Cuiabana: o horário de funcionamento é relevante nas unidades/ laboratório de diagnóstico por imagem e nas unidades de reabilitação, já a qualificação é problemática em todas as unidades consideradas.

Do ponto de vista da demanda (Quadro 4), em apenas uma região - Manaus - não foi destacada a incapacidade de reconhecer o local apropriado para atendimento à saúde. Em todas as demais regiões isso foi considerado como barreira pelos entrevistados.

As barreiras de acesso relacionadas à Disponibilidade foram expressivas em todas as Regiões de Saúde consideradas, constatando-se questões relacionadas a: indisponibilidade de serviços, integração falha ou inexistente entre os serviços, tempo de espera prolongado e insuficiência de medicamentos. Porém, as regiões da Baixada Cuiabana e Manaus se destacam pela maior quantidade de barreiras desse tipo, na percepção de entrevistados.

\section{Aceitabilidade}

Entre as dimensões consideradas, a Aceitabilidade é aquela que aborda o maior número de aspectos relacionados à demanda, como se observa no Quadro 5.

Quanto à oferta, o acolhimento pelos profissionais foi interpretado como a forma de receber os pacientes. No cuidado ao AVC, esta barreira foi destacada em todos os tipos de unidade na Baixada Cuiabana e nas unidades de Emergência na Região de Petrolina/Juazeiro.

A principal barreira apontada no âmbito da demanda foi a crença de não ter problemas de saúde, destacada em todas as cinco regiões do estudo. A preferência por formas alternativas de cuidados é uma importante barreira em três regiões: Baixada Cuiabana, Manaus e Petrolina/Juazeiro. Nessa última, a possível estigmatização (relacionada à preocupação com o que os amigos, colegas de trabalho ou profissionais de saúde possam pensar, dizer ou fazer quando souberem que o usuário procurou a unidade de saúde para receber atendimento) também foi relevante.

Em relação à dimensão Aceitabilidade, destacam-se barreiras nas regiões de Petrolina/Juazeiro e da Baixada Cuiabana. A primeira por registrá-las em todos os aspectos e a segunda por haver barreiras relativas à oferta em todas as unidades consideradas no cuidado ao AVC. 


\section{Quadro 4}

Barreiras de acesso referentes à Disponibilidade, por parte da oferta e da demanda, segundo tipo de barreira e Região de Saúde, 2016.

\begin{tabular}{|c|c|c|c|c|c|}
\hline Barreiras & $\begin{array}{l}\text { Baixada Cuiabana } \\
\text { (Mato Grosso) }\end{array}$ & $\begin{array}{l}\text { Manaus, Entorno e } \\
\text { Alto do Rio Negro } \\
\text { (Amazonas) }\end{array}$ & $\begin{array}{c}\text { Petrolina } \\
\text { (Pernambuco)/ } \\
\text { Juazeiro (Bahia) }\end{array}$ & $\begin{array}{c}\text { Carbonífera/Costa } \\
\text { Doce (Rio Grande } \\
\text { do Sul) }\end{array}$ & $\begin{array}{l}\text { Norte-Barretos/Sul- } \\
\text { Barretos (São Paulo) }\end{array}$ \\
\hline \multicolumn{6}{|l|}{ OFERTA } \\
\hline $\begin{array}{l}\text { Lista de espera com } \\
\text { tempo inadequado às } \\
\text { necessidades }\end{array}$ & Sim & Sim & Sim & Sim & Sim \\
\hline $\begin{array}{l}\text { Tempo de espera } \\
\text { incompatível com } \\
\text { o atendimento de } \\
\text { emergência }\end{array}$ & Sim & Sim & Sim & Sim & Não \\
\hline $\begin{array}{l}\text { Agendamento de serviços * } \\
\text { não é realizado em tempo } \\
\text { oportuno }\end{array}$ & Sim para consultas & $\begin{array}{l}\text { Sim para quase } \\
\text { todos }\end{array}$ & Não para quase todos & Não para quase todos & Não para quase todos \\
\hline $\begin{array}{l}\text { Insuficiência de } \\
\text { medicamentos }\end{array}$ & Não & Não & Sim & Não & Sim \\
\hline $\begin{array}{l}\text { Horário de funcionamento } \\
\text { inadequado em relação ao } \\
\text { cuidado ao AVC }\end{array}$ & $\begin{array}{l}\text { Sim em unidades/ } \\
\text { laboratórios de } \\
\text { diagnóstico por } \\
\text { imagem e de } \\
\text { reabilitação }\end{array}$ & Não & $\begin{array}{l}\text { Sim em unidades/ } \\
\text { laboratórios de } \\
\text { diagnóstico por } \\
\text { imagem }\end{array}$ & Não & Não \\
\hline $\begin{array}{l}\text { Qualificação dos } \\
\text { profissionais no cuidado } \\
\text { ao AVC }\end{array}$ & Sim & $\begin{array}{l}\text { Sim em unidades } \\
\text { de urgência/ } \\
\text { emergência }\end{array}$ & Não & Não & Não \\
\hline \multicolumn{6}{|l|}{ DEMANDA } \\
\hline $\begin{array}{l}\text { Incapacidade de } \\
\text { reconhecer local para } \\
\text { atendimento é barreira }\end{array}$ & Sim & Não & Sim & Sim & Sim \\
\hline
\end{tabular}

AVC: acidente vascular cerebral.

Fonte: estudo de campo da pesquisa Região e Redes - Política, Planejamento e Gestão das Regiões e Redes de Atenção à Saúde no Brasil 14.

* Consultas em cardiologia, em neurologia, em oftalmologia, em endocrinologia, em nefrologia, em nutrição, e em reabilitação; eletrocardiograma, ecocardiograma, exame de fundo de olho, perfil lipídico, mamografia para rastreamento de câncer de mama (mulheres de 50 a 69 anos) e colposcopia para diagnóstico de lesões precursoras de câncer de colo de útero.

\section{Discussão}

A análise das barreiras de acesso nas Regiões de Saúde consideradas no projeto Região e Redes possibilita uma leitura de diversas barreiras em sua diversidade e importância. As desigualdades regionais do país estão expressas nos resultados, já que, em geral, as regiões do Sul e Sudeste registraram menor quantidade e diversidade de barreiras, principalmente considerando a Região de Barretos. Um estudo baseado em dados de 1998 e 2003 da Pesquisa Nacional por Amostra de Domicílios (PNAD) 15 analisou as desigualdades geográficas no acesso aos serviços de saúde no Brasil, considerando o uso de serviços como variável proxy do acesso. Nesse estudo, em ambos os anos analisados, verificou-se que as pessoas residentes nas regiões Sudeste e Sul tiveram maiores chances de uso de serviços de saúde do que os residentes nas demais regiões. Ou seja, da mesma forma que no presente trabalho, em que foi encontrada a menor existência de barreiras de acesso nas regiões Sul e Sudeste, no estudo 


\begin{tabular}{|c|c|c|c|c|c|}
\hline Barreiras & $\begin{array}{c}\text { Baixada } \\
\text { Cuiabana (Mato } \\
\text { Grosso) }\end{array}$ & $\begin{array}{c}\text { Manaus, } \\
\text { Entorno e Alto } \\
\text { do Rio Negro } \\
\text { (Amazonas) }\end{array}$ & $\begin{array}{c}\text { Petrolina } \\
\text { (Pernambuco)/ } \\
\text { Juazeiro (Bahia) }\end{array}$ & $\begin{array}{c}\text { Carbonífera/Costa } \\
\text { Doce (Rio Grande } \\
\text { do Sul) }\end{array}$ & $\begin{array}{c}\text { Norte-Barretos/ } \\
\text { Sul-Barretos } \\
\text { (São Paulo) }\end{array}$ \\
\hline \multicolumn{6}{|l|}{ OFERTA } \\
\hline $\begin{array}{l}\text { Acolhimento de pacientes no } \\
\text { cuidado ao AVC }\end{array}$ & $\begin{array}{l}\text { Sim em todas as } \\
\text { unidades * }\end{array}$ & Não & $\begin{array}{l}\text { Sim em unidades de } \\
\text { emergência }\end{array}$ & Não & Não \\
\hline \multicolumn{6}{|l|}{ DEMANDA } \\
\hline $\begin{array}{l}\text { Preferências por formas alternativas } \\
\text { de cuidados }\end{array}$ & Sim & Sim & Sim & Não & Não \\
\hline $\begin{array}{l}\text { Crença de não ter problema de } \\
\text { saúde }\end{array}$ & Sim & Sim & Sim & Sim & Sim \\
\hline Estigma & Não & Não & Sim & Não & Não \\
\hline
\end{tabular}

AVC: acidente vascular cerebral.

Fonte: estudo de campo da pesquisa Região e Redes - Política, Planejamento e Gestão das Regiões e Redes de Atenção à Saúde no Brasil 14.

* Unidades de urgência e emergência, internação, tratamento intensivo e reabilitação, e laboratórios e unidades de diagnóstico por imagem.

prévio baseado em dados da PNAD as regiões Sul e Sudeste apresentaram maior acesso aos serviços de saúde (avaliado pela variável uso de serviços), apesar das diferenças entre estes estudos, relativas a: tipologia das regiões (Regiões de Saúde vs. macrorregiões); e fonte dos dados (na PNAD, os dados foram obtidos com base em entrevistas com os usuários do sistema de saúde, e neste trabalho as entrevistas foram realizadas com os gestores, prestadores de serviços e profissionais de saúde).

Ocorreu um aumento das desigualdades geográficas no acesso aos serviços de saúde no país no começo do século XXI ${ }^{9}$, e a análise das barreiras de acesso permite destacar persistências dos diferenciais no período mais recente. A insuficiência de recursos e serviços, as diferenças socioeconômicas e a dificuldade de integração entre serviços expressam algumas das disparidades encontradas na realidade do país. Um estudo mais recente 16 analisou as 438 Regiões de Saúde com relação a: transformações socioeconômicas; oferta e complexidade de serviços de saúde, no Brasil, nos períodos de 2000 e 2016. Os autores observaram melhoria nos níveis de renda e oferta de serviços, bem como melhoria nas condições socioeconômicas da população residente nas Regiões de Saúde analisadas. As Regiões de Saúde foram agrupadas de acordo com o grau de desenvolvimento socioeconômico e a oferta e complexidade dos serviços de saúde. Os grupamentos com maiores níveis de desenvolvimento socioeconômico e de oferta de serviços estavam localizados predominantemente nas macrorregiões Sul e Sudeste. No entanto, foram verificadas melhorias entre os períodos analisados que, segundo os autores, podem ser explicadas pela combinação de políticas sociais e econômicas, associadas a estratégias regionais de desenvolvimento, no período analisado 16.

De um modo geral, as desigualdades são expressas por meio da maior relevância do conjunto das barreiras de acesso aos serviços nas Regiões de Saúde da Baixada Cuiabana, Petrolina/Juazeiro e Manaus, localizadas nas regiões menos desenvolvidas do país. As três registraram relevância de todos os tipos de barreiras considerados: Acessibilidade Geográfica, Disponibilidade e Aceitabilidade, já a Região Carbonífera/Costa Doce destacou-se por problemas relacionados à Disponibilidade.

A Acessibilidade Geográfica aos serviços de saúde é uma dimensão fundamental em função dos desafios que podem representar para a população que necessita de atenção à saúde 12,17,18. A grande área territorial das regiões de Manaus e Petrolina/Juazeiro expressa questões relevantes em seus respectivos contextos, podendo representar problemas no caso de emergências 18, mas também em situações que requerem múltiplos cuidados 17. 
O custo dos transportes é uma barreira de acesso nas regiões situadas no Norte, Nordeste e Centro-oeste do país e se relaciona às desigualdades regionais. Dados divulgados pelo Projeto Avaliação do Desempenho do Sistema Saúde (PROADESS) 19 indicam que a Região de Petrolina/Juazeiro tem a renda média per capita mais baixa dentre as consideradas no estudo, e que as regiões da Baixada Cuiabana e Manaus estão entre as mais desiguais de acordo com a razão entre a renda dos 10\% mais ricos e a dos $40 \%$ mais pobres. Piores condições socioeconômicas associadas ou não à desigualdade compõem outro determinante de acesso aos serviços de saúde.

A Disponibilidade apresenta diversas dimensões relevantes quanto às barreiras de acesso, dentre as quais se destaca a questão da suficiência de recursos. Os indicadores do PROADESS 19 permitem comparações com as percepções dos gestores, prestadores e profissionais de saúde. Em todas há uma percepção de insuficiência de médicos, porém, o número de médicos disponíveis ao SUS por mil habitantes, em 2015, revela que as regiões de Manaus, Barretos e Baixada Cuiabana têm valores superiores às respectivas médias estadual, da grande região e nacional. Possivelmente, o fato de os serviços de saúde nessas regiões serem demandados por moradores de outras regiões resulte na percepção da necessidade de mais profissionais. O mesmo ocorre com relação aos leitos hospitalares disponíveis ao SUS.

Ainda de acordo com as informações referidas, a Região Carbonífera/Costa Doce, cuja população demanda serviços da região da capital gaúcha, é caracterizada por quantidades de médicos, enfermeiros e leitos disponíveis ao SUS inferiores às médias estaduais e da Região Sul, sendo ligeiramente superior à média nacional no caso dos médicos. Para além das desigualdades regionais expressas no conjunto das regiões e abordadas por outros autores 15,16, é possível destacar importantes diferenças internas às regiões, revelando que as disparidades no âmbito da saúde se expressam em múltiplas escalas espaciais.

A integração é fundamental no acesso aos serviços, mas principalmente no âmbito da continuidade do cuidado 12,13,20. A integração no sistema de saúde brasileiro é entendida como um desafio na literatura sobre o tema 6,20, o que é corroborado pelos resultados aqui apresentados, principalmente nas regiões de Manaus e Baixada Cuiabana. Merece destaque, também, a ausência, em todas as Regiões de Saúde analisadas, de compartilhamento de responsabilidades e fluxos de informações em tempo oportuno, no cuidado ao AVC, um dos traçadores da pesquisa.

Os problemas vinculados à lista de espera, à insuficiência de medicamentos e, no âmbito da Aceitabilidade por parte dos potenciais usuários, à questão da crença ou percepção de problemas de saúde pela população são generalizados nas regiões e, segundo a literatura, constituem barreiras de acesso também observadas em outros países 12,21.

A análise aqui realizada destacou a relevância e a amplitude do referencial teórico-conceitual utilizado 13 para a análise das barreiras de acesso, o que, conforme reconhecido na literatura 12,21,22, deve subsidiar a proposição de medidas de intervenção nos diferentes contextos. A identificação e análise de barreiras de acesso existentes nas Regiões de Saúde podem contribuir para o processo de regionalização no país, ao subsidiar medidas que ampliem o acesso aos serviços de saúde. Cabe ressaltar que a contribuição do presente trabalho também esteve relacionada à discussão das barreiras de acesso no âmbito das Regiões de Saúde, e não apenas das macrorregiões brasileiras, permitindo uma avaliação do perfil destas regiões. Estudos futuros poderão permitir comparações em relação a esses aspectos de saúde, considerando a distribuição territorial brasileira baseada nas regiões de saúde.

Por último, cabe a importante ressalva de alguns autores 12,13,21,22: a intervenção sobre as barreiras de acesso deve ser pensada para um conjunto delas, e não apenas sobre uma ou mesmo algumas poucas. Isso porque as barreiras e suas consequências se vinculam de múltiplas formas e seu enfrentamento requer levar em conta estas relações. 


\section{Considerações finais}

A análise das barreiras de acesso é fundamental para a compreensão do uso dos serviços de saúde e de suas limitações no país. Os dados apresentados possibilitaram a identificação de barreiras tanto por parte da oferta, como por parte da demanda. Porém, é importante ressaltar que os mesmos se referem a perspectivas de gestores, prestadores e profissionais de saúde, e não de usuários, que são aqueles efetivamente afetados pelas barreiras.

A utilização dos dados citados, à luz de referencial teórico-conceitual sobre a temática, permitiu a análise das barreiras nas Regiões de Saúde consideradas no estudo. As barreiras mais frequentemente observadas nas regiões estudadas foram: em relação à Acessibilidade, o tempo gasto no trajeto no cuidado ao AVC e o custo com o transporte, aparecendo, respectivamente, em 4 e 3 regiões estudadas. No tocante à Disponibilidade, configuraram barreiras em todas as regiões: indisponibilidade de alguns serviços, indisponibilidade de médicos, compartilhamento de responsabilidades e informações em tempo oportuno, e lista de espera em relação a necessidades. Tempo de espera incompatível com o atendimento de emergência e incapacidade de reconhecer o local para atendimento foram apontados como barreiras em 4 das 5 regiões, e fluxos assistenciais não definidos pela CIR, em 3 das 5 regiões estudadas. Em relação à Aceitabilidade, a crença de não ter problemas de saúde foi apontada como barreira também em todas as regiões, e a busca por formas alternativas de cuidado em 3 das 5 .

Embora os resultados encontrados de forma alguma representem o conjunto do país, retratam situações enfrentadas em diferentes cenários territoriais brasileiros, permitindo levantar questões relevantes para as Regiões de Saúde em geral, apontando pistas para outras análises e mesmo intervenções em várias escalas.

\section{Colaboradores}

R. A. D. Oliveira, C. M. R. Duarte, A. L. B. Pavão e F. Viacava contribuíram com a concepção, processamento e análise das informações e redação do artigo, além de sua revisão final.

\section{Informações adicionais}

ORCID: Ricardo Antunes Dantas de Oliveira (00000003-0144-7288); Cristina Maria Rabelais Duarte (0000-0001-6005-9619); Ana Luiza Braz Pavão (0000-0002-4122-1796); Francisco Viacava (00000003-1486-2157).

\section{Agradecimentos}

A pesquisa contou com recursos do Ministério da Ciência, Tecnologia e Inovação e do Ministério da Saúde, por meio da Chamada MCTI/CNPq/CT Saúde/MS/SCTIE/Decit no 41/2013.

\section{Referências}

1. Da Silva SF, organizador. Redes de Atenção à Saúde: desafios da regionalização no SUS. 2a Ed. Campinas: Saberes Editora; 2013.

2. Dourado DDA, Eduardo P, Elias M. Regionalização e dinâmica política do federalismo sanitário brasileiro. Rev Saúde Pública 2011; 45:204-11.

3. Brasil. Decreto no 7.508 , de 28 de junho de 2011. Regulamenta a Lei no 8.080, de 19 de setembro de 1990, para dispor sobre a organização do Sistema Único de Saúde - SUS, o planejamento da saúde, a assistência à saúde e a articulação interfederativa, e dá outras providências. Diário Oficial da União 2011; 29 jun.

4. Lima SAV, Silva MRF, Carvalho EMF, Pessoa EAC, Brito ESV, Braga JPR. Elementos que influenciam o acesso à atenção primária na perspectiva dos profissionais e dos usuários de uma rede de serviços de saúde do Recife. Physis (Rio J.) 2015; 25:635-56.

5. Sousa FOS, Medeiros KR, Gurgel Junior GD, Albuquerque PC. Do normativo à realidade do Sistema Único de Saúde: revelando barreiras de acesso na rede de cuidados assistenciais. Ciênc Saúde Colet 2014; 19:1283-93.

6. Almeida PF, Giovanella L, Mendonça MHM, Escorel S. Desafios à coordenação dos cuidados em saúde: estratégias de integração entre níveis assistenciais em grandes centros urbanos. Cad Saúde Pública 2010; 26:286-98. 
7. McIntyre D, Thiede M, Birch S. Access as a policy-relevant concept in low- and middleincome countries. Health Econ Policy Law 2009; 4(Pt 2):179-93.

8. Sanchez RM, Ciconelli RM. Conceitos de acesso à saúde. Rev Panam Salud Pública 2012; 31:260-8.

9. Travassos C, Martins M. Uma revisão sobre os conceitos de acesso e utilização de serviços de saúde. Cad Saúde Pública 2004; 20 Suppl 2:S190-8.

10. Joint Comission on Accreditation of Healthcare Organizations. The measurement mandate: on the road performance improvement in health care. Chicago: Department of Publications; 1993.

11. Wörz M, Foubister T, Busse R. Access to health care in the EU Member States. Euro Observer 2006; 8:1-4.

12. Peters DH, Garg A, Bloom G, Walker DG, Brieger WR, Rahman MH. Poverty and access to health care in developing countries. Ann N Y Acad Sci 2008; 1136:161-71.

13. Jacobs B, Ir P, Bigdeli M, Annear PL, Damme WV. Addressing access barriers to health services: an analytical framework for selecting appropriate interventions in low-income Asian countries. Health Policy Plan 2012; 27:288-300.

14. Viana AL. Política, Planejamento e Gestão das Regiões e Redes de Atenção à Saúde no Brasil (resumo executivo). http://www.resbr.net.br/apesquisa/resumo-executivo/\#.WNG1MPnyvIU (acessado em 21/Mar/2017).

15. Travassos C, Oliveira E, Viacava F. Desigualdades geográficas e sociais no acesso aos serviços de saúde no Brasil: 1998 e 2003. Ciênc Saúde Colet 2006; 11:975-86.
16. Albuquerque MV, Viana ALA, Lima LD, Ferreira MP, Fusaro ER, Iozzi FL. Desigualdades regionais na saúde. Ciênc Saúde Colet 2017; 22:1055-64.

17. Oliveira EXG, Melo ECP, Pinheiro RJ, Noronha $\mathrm{CP}$, Carvalho MS. Acesso à assistência oncológica: mapeamento dos fluxos origemdestino das internações e dos atendimentos ambulatoriais. O caso do câncer de mama. Cad Saúde Pública 2011; 27:317-26.

18. Almeida WS, Szwarcwald CL. Mortalidade infantil e acesso geográfico ao parto nos municípios brasileiros. Rev Saúde Pública 2012; 46:68-76.

19. Viacava F, Laguardia J, Ugá MAD, Porto S. PROADESS - Avaliação de Desempenho do Sistema de Saúde Brasileiro: indicadores para monitoramento (relatório de pesquisa). http:// www.proadess.icict.fiocruz.br/Relatorio_ Proadess_08-10-2012.pdf (acessado em 30/ Abr/2017).

20. Hartz ZMA, Contandriopoulos A-P. Integralidade da atenção e integração de serviços de saúde: desafios para avaliar a implantação de um "sistema sem muros". Cad Saúde Pública 2004; 20 Suppl 2:S331-6.

21. Jacobs B, Price N. Improving access for the poorest to public sector health services: insights from Kirivong Operational Health District in Cambodia. Health Policy Plan 2006; 21:27-39.

22. Ahmed SM, Petzold M, Kabir ZN, Tomson G. Targeted intervention for the ultra poor in rural Bangladesh: does it make any difference in their health-seeking behaviour? Soc Sci Med 2003; 63:2899-911. 


\section{Abstract}

The study aimed to analyze barriers in access to health services in five Health Regions of Brazil. The study analyzed the answers to semi-structured questionnaires applied to health policymakers, providers, and professionals from the Brazilian Unified National Health System, referenced on the following dimensions: Geographic Accessibility, Availability, and Acceptability. The analysis pointed to specificities in barriers to access in the five regions, while others such as location and time spent to reach the service were barriers in nearly all of them, based on care for stroke patients. In terms of Availability, there were issues related to shortage of physicians, long waiting time, and integration among services in all the regions. As for Acceptability, the study highlighted users' belief that they have no health problems. The Health Regions located in South and Southeast Brazil showed the fewest and least variety of barriers, revealing the persistence of regional inequalities. Although the results do not represent the country as a whole, they allow identifying relevant issues for regionalization of the national health system.

Health Services Accessibility; Regional Health Planning; Health Status Disparities

\section{Resumen}

El objetivo fue analizar las barreras de acceso a los servicios de salud existentes en cinco Regiones de Salud de Brasil. Se analizaron las respuestas obtenidas a partir de la aplicación de cuestionarios semiestructurados a gestores, prestadores $y$, en número más restringido, a profesionales de salud del Sistema Único de Salud, teniendo como referencia las dimensiones: Accesibilidad Geográfica, Disponibilidad y Aceptabilidad. Se verificaron especificidades en relación con las barreras de acceso en las regiones consideradas, mientras otras como la localización y el tiempo invertido en el trayecto, fueron barreras en casi todas ellas, cuando se consideró el cuidado a un accidente vascular cerebral. En cuanto a la Disponibilidad, se observaron cuestiones relacionadas con la insuficiencia de médicos, tiempo de espera prolongado y problemas relativos a la integración, en todas las regiones. En cuanto a la Aceptabilidad, se destacó la creencia de los usuarios de no poseer problemas de salud. Las Regiones de Salud situadas en el Sur y Sudeste del país registraron menor cantidad y diversidad de barreras, revelando la persistencia de desigualdades regionales. A pesar de que los resultados no representen al conjunto del país, permiten apuntar cuestiones relevantes sobre el proceso de regionalización del sistema de salud en el país.

Accesibilidad a los Servicios de Salud;

Regionalización; Disparidades en el Estado de Salud
Recebido em 18/Jun/2018

Versão final reapresentada em 04/Abr/2019

Aprovado em 16/Mai/2019 